\title{
MT-BCS-Based DoA and Bandwidth Estimation of Unknown Signals through Multiple Snapshots Data
}

\author{
Shi Hui Zhang $\mathbb{D}^{\mathbb{D}}$, Qing He Zhang $\mathbb{D}$, Li Ping Shi, Chao Yi $\mathbb{D}$, and Guang Xu Liu $(\mathbb{D}$ \\ The College of Computer and Information, China Three Gorges University, Yichang, China \\ Correspondence should be addressed to Qing He Zhang; zqh@ctgu.edu.cn
}

Received 18 October 2019; Accepted 10 December 2019; Published 6 January 2020

Academic Editor: Chien-Jen Wang

Copyright (c) 2020 Shi Hui Zhang et al. This is an open access article distributed under the Creative Commons Attribution License, which permits unrestricted use, distribution, and reproduction in any medium, provided the original work is properly cited.

\begin{abstract}
The Direction-of-Arrival (DoA) and bandwidth (BW) estimation strategy impinging on a linear array using multiple snapshots data is addressed within the multitask Bayesian Compressive Sensing (MT-BCS). The DoA estimation is used as the reconstruction of sparse signal constrained by the Laplace prior through multitask Bayesian Compressive Sensing. Receiving wideband signal data through linear array, the space is divided into I parts according to the equal interval. The data of interest are assumed to be represented as I-dimensional vector, and the wideband signal can be reconstructed accurately using only a small number $M$. The receiving antenna operates in the frequency range $\left[f_{\min }, f_{\max }\right]$. Starting from the voltages measured at the output of the array elements at a multiple time instants at $f_{p}=f_{\min }+\Delta f, p=1, \ldots, P$, the retrieval of the DoAs is addressed by means of a customized strategy based on MT-BCS in order to correlate the solutions obtained over different frequency samples. The bandwidth of the signals is obtained as a byproduct by identifying at which frequencies the MT-BCS estimations include a signal along the $i$ th $(i=1, \ldots, I)$ sampling direction. From the outputs of different frequencies, we can know the DoA and BW of signals. A preliminary numerical result is reported to show the behavior of the proposed approach in multiple snapshots data.
\end{abstract}

\section{Introduction}

Direction-of-Arrival (DoA) estimation has important applications in many traditional fields such as smart antenna array, radar signal processing, geophysical or seismic sensing, acoustics, multiple-input multiple-output (MIMO), and other applications related to finding the direction of the incoming signals or sources [1-4]. In fact, the DoAs knowledge of receiving signal can be properly used to locate the corresponding source, and the adaptive beamforming of the receiving antenna pattern can improve the sensitivity of the system to the desired signal direction or suppress unnecessary interference.

In some scientific literature, several effective methodologies have been proposed about DoA estimation, such as the multiple signal classification (MUSIC) [5] and the signal estimation parameter via rotational invariance technique (ESPRIT) [6]. For the limitations that the need of a priori knowing, the number of incoming signals and the calculation of the correlation matrix are usually computationally expensive, and their standard implementations are rarely available especially nowadays with the huge proliferation of wireless devices/services and the presence of noncollaborative users [7]. More recently, DoA estimation strategies based on the Compressive Sensing (CS) $[8,9]$ are proposed and have shown promising features and results. CS is an enabling paradigm for many applications where there is the need of overcoming the Shannon's limit in data acquisition and to recover sparse signals from far fewer measurements [10]. Thanks to these features that the computational efficiency, the accuracy, and the robustness to the noise, CS-based strategies have already been applied to a variety of applications in electromagnetic engineering $[11,12]$. But for guaranteeing reliable estimations, the sampling matrix must satisfy the restricted isometry property (RIP) [13] when applying CS. Unfortunately, such a condition cannot be easily verified because of it results are computationally demanding [14]. But innovative approaches using the Bayesian compressive sensing (BCS) $[15,16]$ have been proposed, and they have improved this 
problem. However, most of the works about DoA estimation are mainly focused on narrowband signals. In [17], the narrowband algorithm is simply extended to broadband. As for the DoAs estimation of wideband signals, because of the guiding vector of broadband signal frequency, the signal space of different sub-bands is inconsistent, which makes it difficult to separate the signal space from noise space. General, subspace-based estimation techniques [18] based on the root-MUSIC [19] and maximum likelihood [20] are used to estimate the DoAs of the wideband signal, but they also have limitations. This problem has been shown in $[21,22]$, where the MT-BCS [23] has been customized to deal with wideband signals while exploiting the correlation among different frequency samples taken from single snapshot data and multiple snapshots, respectively.

In this paper, the DoAs and BW estimation problem of wideband signals are formulated within the MT-BCS framework based on Laplace priors. Starting from the key observation that the wideband signal affecting the antenna array is essentially sparse in spatial domain. Because the signal is a wideband signal, we sample the signal with a certain range of frequencies in order to estimate the bandwidth of the signal. Set the sampling frequency range to $\left[f_{\min }, f_{\max }\right]$, the DoAs and BW are estimated at the same time by which the range of frequencies has measured data.

The rest of the paper is organized as follows. The Bayesian modeling is mathematically formulated in Section 2, where the wideband signal model of MT-BCS using Laplace priors is described. A set of representative numerical results is then reported and discussed in Section 3, where reference DoAs and bandwidth estimation methods are performed. Finally, some conclusions are drawn in Section 4.

\section{Bayesian Modeling}

CS theory can cover certain signals from far fewer samples or measurements than traditional methods, and it is a very important step to restore the signal we want. In some work, narrowband signals are recovered by MT-BCS $[24,25]$. In this section, a simple MT-BCS model about wideband signals based on Laplace priors is shown.

2.1. Model for BCS. Let us consider a set of $K$ wideband signals impinging on a planar distribution of $N$ sensors is located. The DoAs of the signals are denoted as $\theta_{k}, k=1, \ldots, K$, and operating in the frequency range $\left[f_{\min }, f_{\max }\right]$. The voltages collected from the output of the array elements at $P$ different frequency samples $f_{p}=f_{\min }+$ $\Delta f(2 p-1) / 2, p=1, \ldots, P, \quad$ being $\Delta f=\left(f_{\max }-f_{\text {min }}\right)$ $/(P-1)$. The input data of the problem are the voltages $v_{n}^{(p)}, n=1, \ldots, N$, measured by each sensor expressed as

$$
v_{n}^{(p)}=\sum_{k=1}^{K} s_{k} \hat{y} \cdot h e^{j\left(2 \pi / \lambda^{(p)}\right) x_{n} \sin \theta_{k}}+\eta_{n}^{(p)}, \quad n=1, \ldots, N,
$$

where $s_{k}, k=1, \ldots, K$, is the amplitude of the impinging signals, $h$ is the antenna effective length, $\lambda^{(p)}$ being the free- space length at $p$ th frequency, and $\eta_{n}^{(p)}, n=1, \ldots, N$, the contribution of the noise at each sensor.

Then, in order to employ the proposed MTBCS-based methodology, the problem is reformulated by sampling the angular domain of interest over a very fine grid of $I>>K$ angular location, being $\theta_{i}=-(\pi / 2)+\pi(i-1) /(I-1)$, $i=1,2, \ldots, I$. Under this assumption and considering the multiple-snapshots case, (1) can be rewritten in a matrix form as

$$
\mathbf{v}_{l}^{(p)}=\boldsymbol{\Phi}^{(p)}(\boldsymbol{\theta}) \mathbf{s}_{l}^{(p)}+\boldsymbol{\eta}_{l}^{p}, \quad l=1, \ldots, L,
$$

where $L$ is the number of snapshots, $\mathbf{s}_{l}^{(p)}$ is the vector of estimated signals, $\boldsymbol{\Phi}^{(p)}(\boldsymbol{\theta})=\boldsymbol{\Psi} \mathbf{A}^{(p)}(\boldsymbol{\theta}), \boldsymbol{\Psi} \in \mathbf{R}^{\mathbf{M} \times \mathbf{N}}$, is the measurement matrix, we will use the Gaussian matrix in this paper, and $\mathbf{M}(\mathbf{M}<<I)$ is the number of samples [16]. $\mathbf{A}^{(p)}$ is the matrix of array manifold:

$\mathbf{A}^{(p)}(\boldsymbol{\theta})=\left[\begin{array}{cccc}e^{j\left(2 \pi / \lambda^{(p)}\right) x_{1} \sin \theta_{1}} & e^{j\left(2 \pi / \lambda^{(p)}\right) x_{1} \sin \theta_{2}} & \cdots & e^{j\left(2 \pi / \lambda^{(p)}\right) x_{I} \sin \theta_{1}} \\ e^{j\left(2 \pi / \lambda^{(p)}\right) x_{2} \sin \theta_{1}} & e^{j\left(2 \pi / \lambda^{(p)}\right) x_{2} \sin \theta_{2}} & \cdots & e^{j\left(2 \pi / \lambda^{(p)}\right) x_{2} \sin \theta_{I}} \\ \vdots & \vdots & \ddots & \vdots \\ e^{j\left(2 \pi / \lambda^{(p)}\right) x_{N} \sin \theta_{1}} & e^{j\left(2 \pi / \lambda^{(p)}\right) x_{N} \sin \theta_{2}} & \cdots & e^{j\left(2 \pi / \lambda^{(p)}\right) x_{N} \sin \theta_{I}}\end{array}\right]$,

where $x_{n}=(n-1) d, n=1, \ldots, N$, is the distance between the $i$ th array element and the reference array element and $d$ is the array element spacing.

2.2. Model for MT-BCS Based on Laplace Priors. In [15], we can learn about the general model of MT-BCS based on Laplace priors. So, this section of the model simply recounts the expressions of the wideband signals we want to use. Specify sparse signal $\mathbf{s}_{l}^{(p)}$ as a Laplace priors about parameter $\lambda$, and it is defined as

$$
p\left(\mathbf{s}_{l}^{(p)} \mid \lambda\right)=\frac{\lambda}{2} \exp \left(-\frac{\lambda}{2}\left\|\mathbf{s}_{l}^{(p)}\right\|_{1}\right) .
$$

And the mean and covariance of signals are given by

$$
\begin{aligned}
& \mu=\beta \Sigma \boldsymbol{\Phi}^{T} \mathbf{v}_{l}^{(p)}, \\
& \Sigma=\left[\beta \boldsymbol{\Phi}^{T} \boldsymbol{\Phi}+\boldsymbol{\Lambda}\right]^{-1},
\end{aligned}
$$

where $\boldsymbol{\Lambda}=\operatorname{diag}\left(1 / \gamma_{i}\right), i=1, \ldots, I, \gamma_{i}$, is the signal hyperparameter and estimated as

$$
\gamma_{i}= \begin{cases}\frac{-s_{i}\left(s_{i}+2 \lambda\right)+s_{i} \sqrt{\left(s_{i}+2 \lambda\right)^{2}-4 \lambda\left(s_{i}-q_{i}^{2}+\lambda\right)}}{2 \lambda s_{i}^{2}}, & \text { if } q_{i}^{2}-s_{i}>\lambda, \\ 0, & \text { otherwise, }\end{cases}
$$

with

$$
\begin{gathered}
s_{i}=\frac{S_{i}}{1-\gamma_{i} S_{i}}, \\
q_{i}=\frac{Q_{i}}{1-\gamma_{i} S_{i}},
\end{gathered}
$$




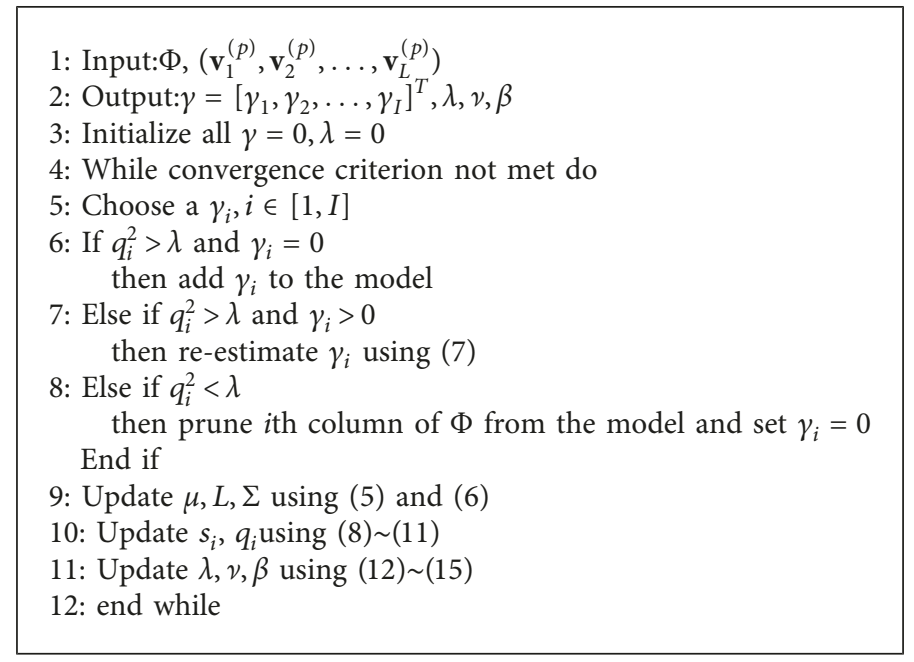

Algorithm 1: Algorithm of MT-BCS.

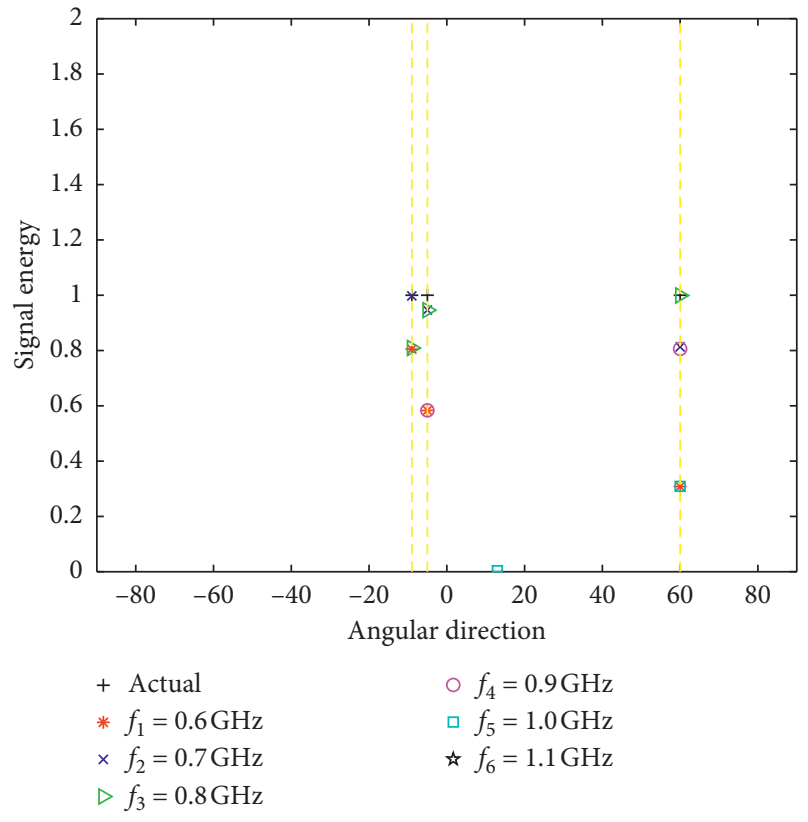

Figure 1: Actual and estimated DoAs for multiple snapshots.

TABle 1: Estimated DoAs and signal energy for different frequencies.

\begin{tabular}{lcc}
\hline & $\widetilde{\theta}$ & $\widetilde{s}$ \\
\hline$f_{1}$ & $(-9,-5,60)$ & $(0.8029,0.5749,0.3021)$ \\
$f_{2}$ & $(-9,-5,60)$ & $(1.0005,0.9356,0.8111)$ \\
$f_{3}$ & $(-9,-5,60)$ & $(0.8117,0.9323,0.9916)$ \\
$f_{4}$ & $(-5,44,60)$ & $(0.5714,-0.0070,0.7933)$ \\
$f_{5}$ & $(13,39,60)$ & $(0.0212,-0.0048,0.2998)$ \\
$f_{6}$ & $(-9,-5,60)$ & $(0.0044,-0.0021,0.0015)$ \\
\hline
\end{tabular}

$$
\begin{gathered}
S_{i}=\beta \phi_{i}^{T} \phi_{i}-\beta^{2} \phi_{i}^{T} \boldsymbol{\Phi} \Sigma \boldsymbol{\Phi}^{T} \phi_{i}, \\
Q_{i}=\beta \phi_{i}^{T} \mathbf{v}_{l}^{(p)}-\beta^{2} \phi_{i}^{T} \boldsymbol{\Phi} \Sigma \boldsymbol{\Phi}^{T} \mathbf{v}_{l}^{(p)},
\end{gathered}
$$

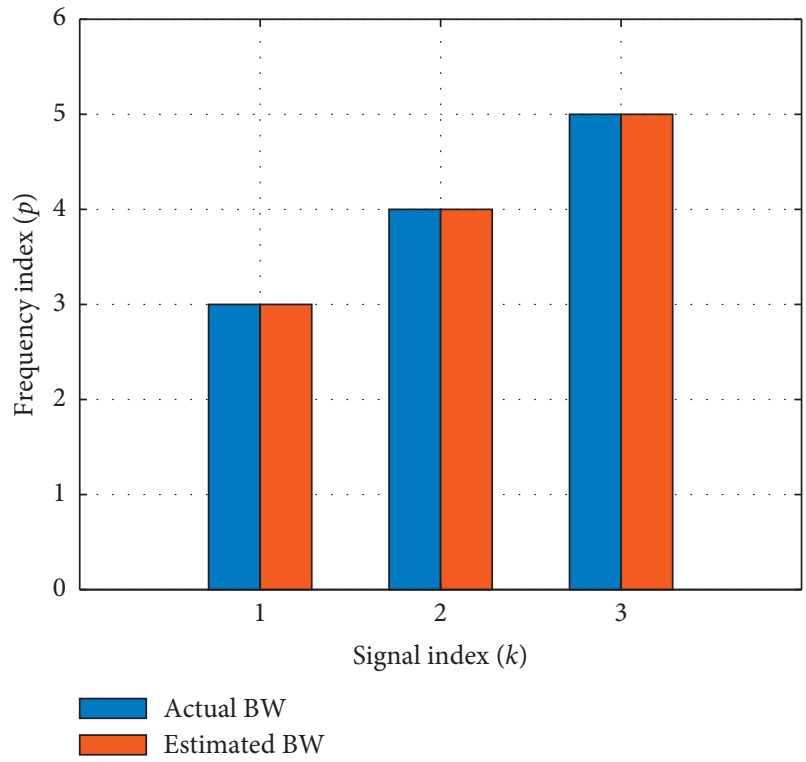

FIgURe 2: Actual and estimated signals bandwidth.

where $\phi_{i}, i=1, \ldots, I$, expresses the $i$ th column of $\Phi, \beta$ is the noise hyperparameter and Gamma prior obeying parameters $a$ and $b$. Parameter $\lambda$ realizes the following Gamma hyperprior:

$$
p(\lambda \mid \nu)=\operatorname{Gamma}(\lambda \mid \nu / 2, \nu / 2)(10) .
$$

The parameters estimation of $\lambda, \beta$, and $v$ is expressed as following:

$$
\begin{aligned}
& \lambda=\frac{N-1+v / 2}{\sum_{i=1}^{N}\left(\gamma_{i} / 2\right)+v / 2}, \\
& \beta=\frac{\mathrm{LN} / 2+a}{\sum_{l=1}^{L}\left(\left\langle\left\|\mathbf{v}_{l}^{(p)}-\Phi \mathbf{s}_{l}^{(p)}\right\|^{2}\right\rangle / 2\right)+b},
\end{aligned}
$$




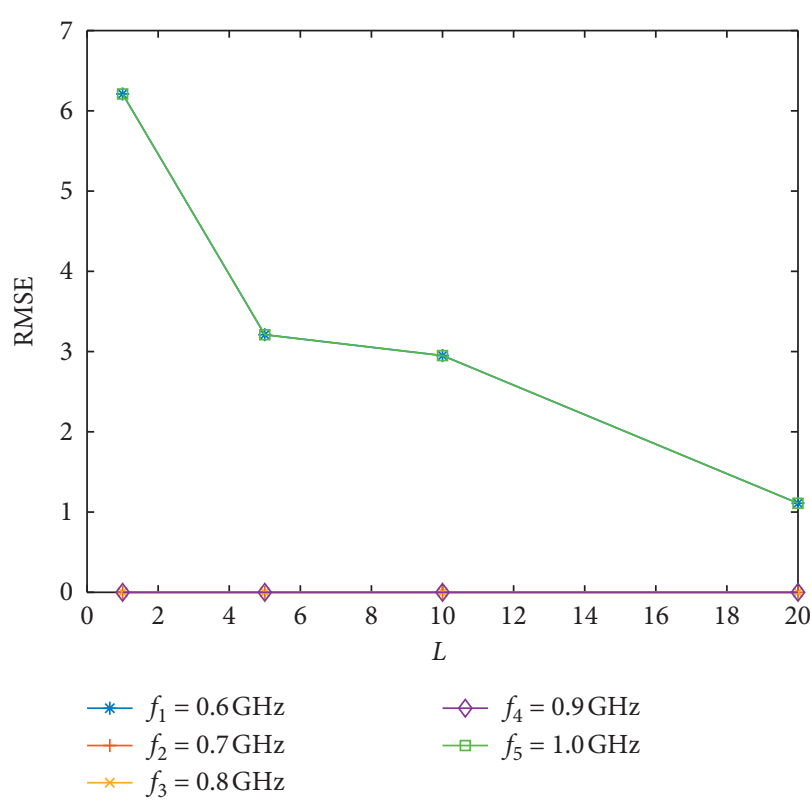

(a)

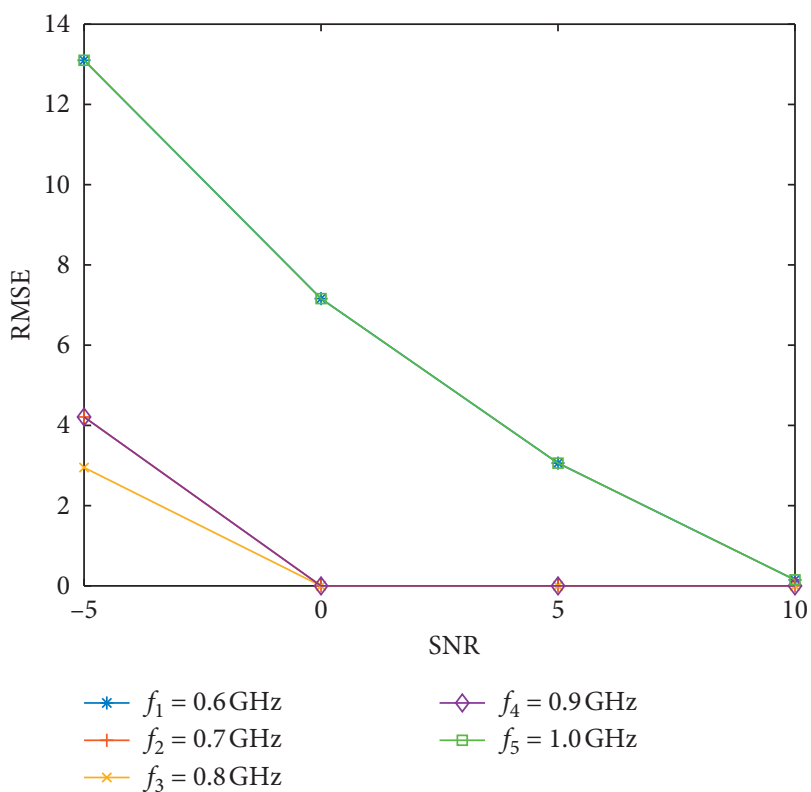

(c)

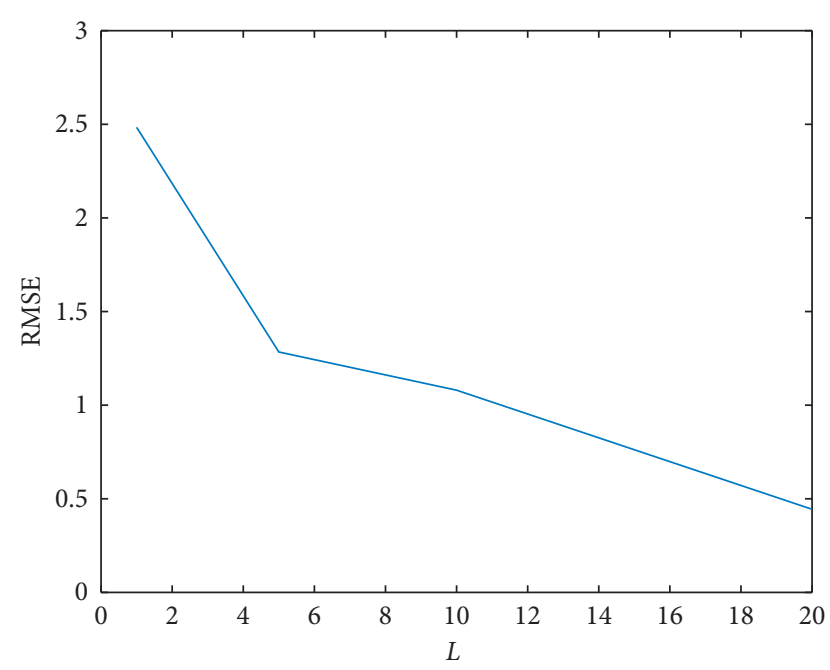

(b)

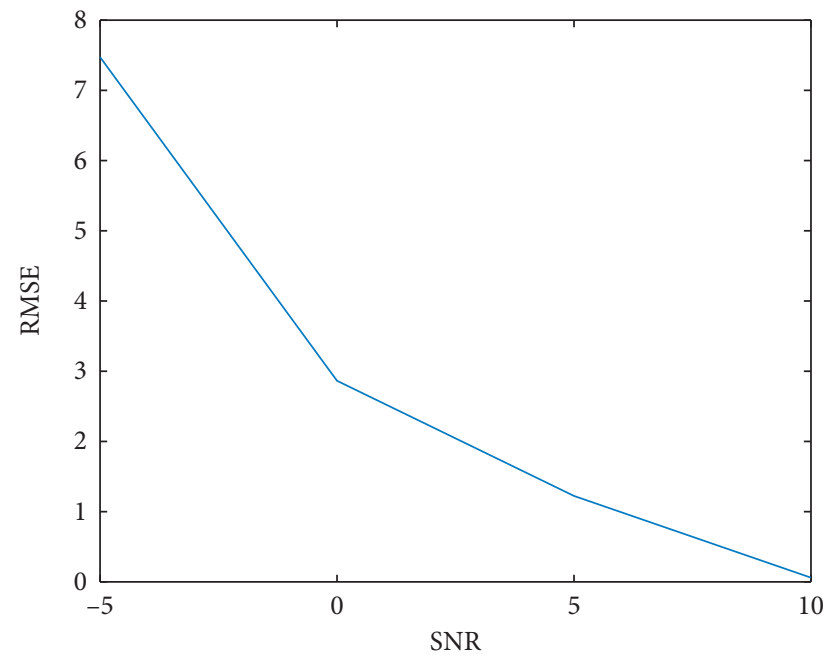

Figure 3: Root mean square error (RMSE) versus L/SNR. (a) Behavior of RMSE versus $L$ for different frequencies of $f_{p}, p=1, \ldots, 5$. (b) Behavior of average RMSE versus SNR; set SNR $=10$. (c) Behavior of RMSE versus SNR for different frequencies of $f_{p}, p=1, \ldots, 5$. (d) Behavior of average RMSE versus SNR; set $L=20$.

$$
\ln (v / 2)+1-\psi(v / 2)+\ln \lambda-\lambda=0 .
$$

From the above formula, we can see that it is known that $\gamma, \lambda, \beta$, and $\nu$ can find $\mu$ and $\Sigma$ using (5) and (6). It is known that $\mu$ and $\Sigma$ can also find $\gamma, \lambda, \beta$, and $\nu$ using (7) (15). The procedure is summarized in Algorithm 1.

\section{Numerical Results}

In this section, we present experimental results that demonstrate the performance of MT-BCS based on
Laplace priors to recovery wideband signals. Different from narrow narrowband signals, the wideband signal exists only when sampling frequency $f_{p}$ is within the range of bandwidth.

We use the following default setup in the experimental results reported in this section. Signal matrix of length $I$ is generated, where $K$ coefficients are located at signal randomly, and the rest $(I-K)$ of the coefficients are set equal to zero. As the measurement matrix $\Psi$, we chose a Gaussian matrix, where the columns $\psi_{i}$ are Gaussian distributed on the sphere $R^{I}$. Moreover, we present results with noisy 


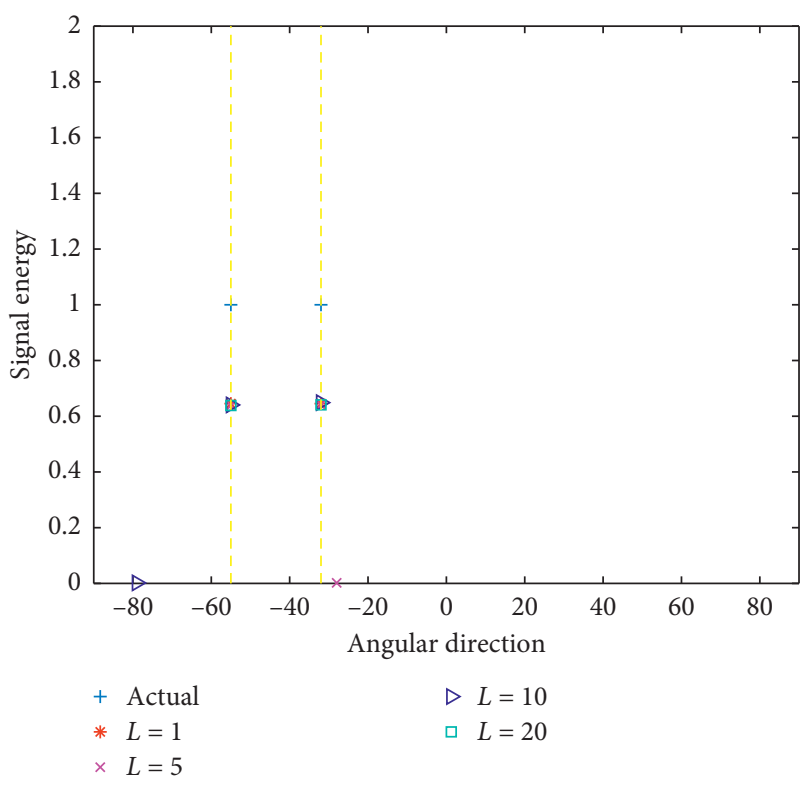

Figure 4: Actual and estimated DoAs for average by (18).

TABle 2: The estimated DoAs for frequencies verse snapshots.

\begin{tabular}{ccccc}
\hline \multicolumn{5}{c}{$\tilde{\theta}$} \\
& $L=1$ & $L=5$ & $L=10$ & $L=20$ \\
\hline$f_{1}$ & $(-55,-32,10)$ & $(-55,-32,12)$ & $(-55,-32,12)$ & $(-55,-49$, \\
& $(-55,-32$, & $(-56,-55$, & $(-55,-32)$ & $(-55,-32)$ \\
$f_{2}$ & $-23)$ & $-32)$ & $(-55,-32)$ & $(-55,-32)$ \\
$f_{3}$ & $(-55,-32,26)$ & $(-55,-32)$ & $(-55,-32)$ & $(-55,-32)$ \\
$f_{4}$ & $(-55,-32)$ & $(-55,-32)$ & $(-52)$ \\
$f_{5}$ & $(-55,-32$, & $(-55,-32$, & $(-79,-55$, & $(-55,-32,48)$ \\
\hline
\end{tabular}

acquisitions, where for the noisy observations we add white Gaussian noise.

In the first set of experiments, let us consider $K=3$ signals arriving on a linear array of $N=30$ elements, half wavelength spaced at $f_{0}=\left(f_{\min }+f_{\max }\right) / 2$, and designed to work in the frequency range $\left[f_{\min }, f_{\max }\right]=[0.6: 1.1] \mathrm{GHz}$. The actual signals are characterized by a sinusoidal wave with amplitude $s_{k}=+1, k=1, \ldots, K$, DoAs equal to $\theta_{1}=-9 \mathrm{deg}, \theta_{2}=-5 \mathrm{deg}$, and $\theta_{3}=60 \mathrm{deg}$, and bandwidths $b_{1}=[0.6: 0.8] \mathrm{GHz}, \quad b_{2}=[0.6: 0.9] \mathrm{GHz}, \quad$ and $b_{3}=[0.6: 1.0] \mathrm{GHz}$. In order to estimate the DoAs and the signal bandwidth by means of the MT-BCS, the measured data are considered characterized by a signal-to-noise ratio equal to $\mathrm{SNR}=10 \mathrm{~dB}$ and are sampled at $P=6$ frequencies $\left(f_{1}, f_{2}, f_{3}, f_{4}, f_{5}, f_{6}\right)=(0.6,0.7,0.8,0.9,1.0,1.1) \mathrm{GHz}$, being $\Delta f=0.1 \mathrm{GHz}$. Set snapshots $L=10$ and the resulted data of $p$ th frequency in multiple snapshots is calculated by

$$
\widetilde{s_{\text {avg }}^{p}}=\sum_{l=1}^{L} \widetilde{s_{l}^{p}} / L,
$$

where $\widetilde{s_{l}^{p}}$ are the estimated signal energy in $l$ th snapshot at $p$ th frequency sampling. Moreover, the angular grid has
TABLE 3: The estimated signal energy.

\begin{tabular}{|c|c|c|c|c|}
\hline & & $\widetilde{s}$ & & \\
\hline & $L=1$ & $L=5$ & $L=10$ & $L=20$ \\
\hline$f_{1}$ & $\begin{array}{c}(0.2934,0.3126 \\
-0.016)\end{array}$ & $\begin{array}{c}(0.2935, \\
0.3044 \\
-0.0134)\end{array}$ & $\begin{array}{c}(0.2935 \\
0.3144 \\
-0.0133)\end{array}$ & $\begin{array}{c}(0.2920 \\
-0.0087 \\
0.3136)\end{array}$ \\
\hline$f_{2}$ & $\begin{array}{c}(0.8098,0.8065 \\
-0.205)\end{array}$ & $\begin{array}{c}(-0.0087 \\
0.8014 \\
0.8189)\end{array}$ & $\begin{array}{l}(0.8090 \\
0.8349)\end{array}$ & $\begin{array}{l}(0.8029 \\
0.7948)\end{array}$ \\
\hline$f_{3}$ & $\begin{array}{c}(0.9969,0.9979 \\
0.0296)\end{array}$ & $\begin{array}{l}(0.9938 \\
0.9857)\end{array}$ & $\begin{array}{l}(0.9978 \\
1.0013)\end{array}$ & $\begin{array}{l}(0.9879 \\
0.9796)\end{array}$ \\
\hline$f_{4}$ & $(0.8068,0.8045)$ & $\begin{array}{l}(0.8014 \\
0.8012)\end{array}$ & $\begin{array}{l}(0.7969, \\
0.7886)\end{array}$ & $\begin{array}{l}(0.8081, \\
0.8057)\end{array}$ \\
\hline$f_{5}$ & $\begin{array}{c}(0.3197,0.3047 \\
-0.0317)\end{array}$ & $\begin{array}{l}(0.2965 \\
0.2897 \\
0.0098)\end{array}$ & $\begin{array}{l}(0.0093, \\
0.3059 \\
0.3050)\end{array}$ & $\begin{array}{c}(0.3018, \\
0.3106 \\
-0.0033)\end{array}$ \\
\hline$\widetilde{s_{\mathrm{avg}}}$ & $\begin{array}{c}(0.6453,0.64524 \\
-0.041,0.0063 \\
-0.0032,0.0059)\end{array}$ & $\begin{array}{l}(0.0017 \\
0.5379 \\
0.6399 \\
0.0020 \\
0.0027)\end{array}$ & $\begin{array}{l}(0.0019 \\
0.6406 \\
0.6488, \\
0.0027)\end{array}$ & $\begin{array}{l}(0.6385, \\
0.0017 \\
0.6409 \\
0.0007)\end{array}$ \\
\hline
\end{tabular}

been discretized with $I=181$ samples in order to obtain a resolution of $\Delta \theta=1 \mathrm{deg}$.

The actual DoAs and those estimated values by the proposed spectral correlation MT-BCS based strategy are shown in Figure 1 and Table 1. As it can be observed in Table 1, there are some values that are small enough to be ignored. This is mainly caused by noise. We ignore these values below 0.1. It can be observed that DoA $\theta_{1}=-9 \mathrm{deg}$ and bandwidth $b_{1}=\left[f_{1}: f_{3}\right]$ for $s_{1}, \theta_{2}=-5 \mathrm{deg}$ and $b_{2}=$ $\left[f_{1}: f_{4}\right]$ for $s_{2}$, and $\theta_{3}=60 \mathrm{deg}$ and $b_{3}=\left[f_{1}: f_{5}\right]$ for $s_{3}$, which is shown Figure 1 and Table 1.

The actual and estimated bandwidths of the $K=3 \mathrm{im}$ pinging signals are shown in Figure 2. It is possible to observe that both the directions of the incoming signals are correctly retrieved (Figure 1) as well as their band widths.

Then, we will see reconstruction error by RMSE. The equation of RMSE is expressed as follows:

$$
\text { RMSE }=\sum_{p=1}^{P} \sum_{k=1}^{K} \sqrt{\sum_{t=1}^{T}\left(\frac{1}{L} \sum_{l=1}^{L} \widehat{\theta}_{f_{p}, k, t, l}-\theta_{k}\right)^{2}} / \mathrm{PK},
$$

where $T$ is number of experiments and $\widehat{\theta}_{f_{p}, k, t, l}$ is the estimated value of $k$ th signal at $p$ th frequency.

In the second set of experiments, let us consider $K=2$ signals, designed to work in the frequency range $\left[f_{\min }, f_{\max }\right]=[0.6: 1.0] \mathrm{GHz}$. DoAs equal to $\theta_{1}=-55 \mathrm{deg}$ and $\theta_{2}=-32 \mathrm{deg}$, and in order to facilitate the use of RMSE calculation error, we set the two signals which have the same bandwidths $[0.6: 1.0] \mathrm{GHz}$. The measured data are sampled at $P=5$ frequencies $\left(f_{1}, f_{2}, f_{3}, f_{4}, f_{5}\right)=(0.6,0.7,0.8,0.9$, 1.0) $\mathrm{GHz}$, being $\Delta f=0.1 \mathrm{GHz}$. Average reconstruction errors of $T=500$ runs are shown for the case in Figure 3 for all types of signals.

It is noted that RMSE results in lower values than setting snapshots minor from Figure 3(b) and the frequency close to $f_{0}$ show better performance than others from Figure 3(a). 


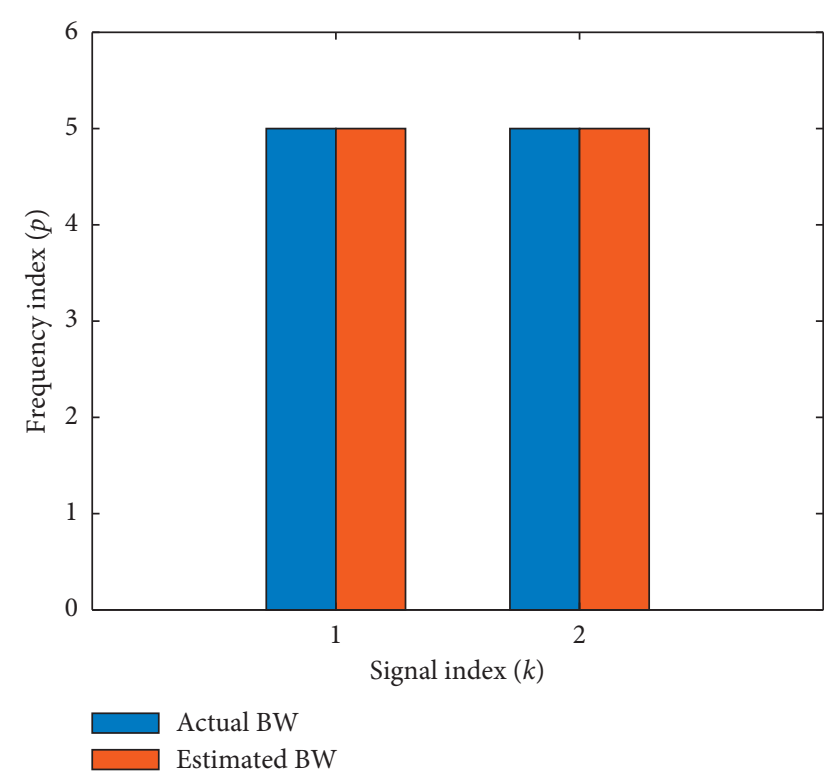

Figure 5: Actual and estimated signal bandwidth.

The results of the same experimental setup with different SNR are shown in Figures 3(c) and 3(d). Similar performance decrease by the increase of SNR can be observed. These suggest that the estimating DoA results can get lower reconstruction error in multiple snapshots and higher SNR. For wideband signals, different sampling frequencies can obtain different reconstruction results. This will have an impact on the accuracy of DoA estimation results.

In order to obtain better reconstruction performances, we will add signal energy $\widehat{s_{\mathrm{avg}}^{p}}$ in (16) at different frequencies of the same signal and average it, and then get $\widetilde{s_{\text {avg }}}$. (16) can be rewritten as follows:

$$
\widetilde{s_{\mathrm{avg}}}=\sum_{p=1}^{P} \sum_{l=1}^{L} \frac{\widetilde{s_{l}^{(p)}}}{\mathrm{LP}} .
$$

In Figure 4, the reconstruction DoA results using (18) at different snapshots are shown. We have also given estimated DoAs and values at pth frequency, as shown in Table 2. $\widetilde{s_{\mathrm{avg}}}$ is the average signal energy of all estimated angles from low to high. The signal energy that shows the unrelated angle is much lower than what we get in the first set experiments. This shows that using (18) to estimate DoA can get better reconstruction results than using (16). We also can note that as the number of snapshots increases, unrelated angles no longer exist. Frequency away from $f_{0}$ still has poor reconstruction results, but this is an improvement after the signal energy of all frequencies is averaged, that is, the signal energy of the unrelated angle becomes smaller.

We ignore values less than 0.1 as in the first set of experiments. It can be concluded that the DoAs and bandwidths of signals form the result shown in Figure 4 and Tables 2 and $3, \theta_{1}=-55 \mathrm{deg}$ and $b_{1}=\left[f_{1}: f_{5}\right]$ for $s_{1}$ and $\theta_{2}=-32 \mathrm{deg}$ and $b_{2}=\left[f_{1}: f_{5}\right]$ for $s_{2}$. The estimated result of signal bandwidths is shown in Figure 5.

\section{Conclusion}

In this paper, we formulated the DoAs and BW-estimated problem of the wideband signal using MT-BCS based on Laplace priors and presented a framework for multiple snapshots data. Using this framework, we first get the actual and estimated DoAs for multiple snapshots at different frequencies. At the same time, the signal BW is obtained by observing at which frequency sampling point there is a signal energy after ignoring values that are small enough. Then, we use the RMSE to evaluate the reconstruction results in different snapshots and SNRs, finding with the increasing of snapshots or SNR, the reconstruction results are better.

\section{Data Availability}

The data used to support the findings of this study are available from the corresponding author upon request.

\section{Conflicts of Interest}

The authors declare that they have no conflicts of interest.

\section{Acknowledgments}

This work was supported by the National Natural Science Foundation of China under Grant no. 61771008.

\section{References}

[1] P. Stoica, P. Babu, and J. Li, "SPICE: a sparse covariance-based estimation method for array processing," IEEE Transactions on Signal Processing, vol. 59, no. 2, pp. 629-638, 2011.

[2] F. Liu, J. Wang, C. Sun, and R. Du, "Spatial differencing method for DOA estimation under the coexistence of both uncorrelated and coherent signals," IEEE Transactions on Antennas and Propagation, vol. 60, no. 4, pp. 2052-2062, 2012.

[3] C. H Niow and H. T. Hui, "Improved noise modeling with mutual coupling in receiving antenna arrays for direction-ofarrival estimation," IEEE Transactions on Wireless Communications, vol. 11, no. 4, pp. 1616-1621, Apr. 2012.

[4] F. Wen, J. Shi, and Z. Zhang, "Joint 2D-DOD, 2D-DOA and polarization angles estimation for bistatic EMVS-MIMO radar via PARAFAC analysis," IEEE Transactions on Vehicular Technology, 2019.

[5] R. Schmidt, "Multiple emitter location and signal parameter estimation," IEEE Transactions on Antennas and Propagation, vol. 34, no. 3, pp. 276-280, 1986.

[6] R. Roy and T. Kailath, "ESPRIT-Estimation of signal parameters via rotational invariance techniques," IEEE Transactions on Acoustics, Speech, and Signal Processing, vol. 37, no. 7, pp. 984-995, 1989.

[7] M. Carlin, P. Rocca, G. Oliveri, F. Viani, and A. Massa, "Directions-of-arrival estimation through bayesian compressive sensing strategies," IEEE Transactions on Antennas and Propagation, vol. 61, no. 7, pp. 3828-3838, 2013.

[8] E. J. Candes and M. B. Wakin, "An introduction to compressive sampling," IEEE Signal Processing Magazine, vol. 25, no. 2, pp. 21-30, 2008.

[9] S. Ji, Y. Xue, and L. Carin, "Bayesian compressive sensing," IEEE Transactions on Signal Processing, vol. 56, no. 6, pp. 2346-2356, 2008. 
[10] R. G. Baraniuk, "More is less: signal processing and the data deluge," Science, vol. 331, no. 6018, pp. 717-719, 2011.

[11] W. L. Chan, M. L. Moravec, R. G. Baraniuk, and D. M. Mittleman, "Terahertz imaging with compressed sensing and phase retrieval," Optics Letters, vol. 33, no. 9, pp. 974-976, 2008.

[12] L. C. Potter, E. Ertin, J. T. Parker, and M. Cetin, "Sparsity and compressed sensing in radar imaging," Proceedings of the IEEE, vol. 98, no. 6, pp. 1006-1020, 2010.

[13] R. Baraniuk, "Compressive sensing [lecture notes]," IEEE Signal Processing Magazine, vol. 24, no. 4, pp. 118-121, 2007.

[14] A. Massa, P. Rocca, and G. Oliveri, "Compressive sensing in electromagnetics-a review," IEEE Antennas and Propagation Magazine, vol. 57, no. 1, pp. 224-238, 2015.

[15] M. Lustig, D. L. Donoho, J. M. Santos, and J. M. Pauly, "Compressed sensing MRI," IEEE Signal Processing Magazine, vol. 25 , no. 2, pp. 72-82, 2008.

[16] S. D. Babacan, R. Molina, and A. K. Katsaggelos, "Bayesian compressive sensing using Laplace Priors," IEEE Transactions on Image Processing, vol. 19, no. 1, pp. 53-63, 2010.

[17] D. L. Donoho, "Compressed sensing," IEEE Transactions on Information Theory, vol. 52, no. 4, pp. 1289-1306, 2006.

[18] Z. D. Lei, X. K. Huang, and S. J. Zhang, "A fast algorithm for direction of arrival estimation of multiple wide-band sources," Journal of the China Railway Society, vol. 19, no. 4, pp. 46-50, 1997.

[19] B. Ottersten and T. Kailath, "Direction-of-arrival estimation for wide-band signals using the ESPRIT algorithm," IEEE Transactions on Acoustics, Speech, and Signal Processing, vol. 38, no. 2, pp. 317-327, 1990.

[20] A. B. Gershman and M. G. Amin, "Coherent wideband DOA estimation of multiple FM signals using spatial time-frequency distributions," in Proceedings of the 2000 IEEE International Conference on Acoustics, Speech, and Signal Processing, pp. 3065-3068, Istanbul, Turkey, June 2000.

[21] M. A. Hannan, P. Rocca, and A. Massa, "Robust BCS-based Direction-of-Arrival and bandwidth estimation of unknown signals for cognitive radar," in Proceedings of the 2018 IEEE International Symposium on Antennas and Propagation \& USNC/URSI National Radio Science Meeting, Boston, MA, USA, July 2018.

[22] S. Ji, D. Dunson, and L. Carin, "Multitask compressive sensing," IEEE Transactions on Signal Processing, vol. 57, no. 1, pp. 92-106, 2009.

[23] M. A. Hannan, N. Anselmi, G. Oliveri, and P. Rocca, "Joint DoA and bandwidth estimation of unknown signals through single snapshot data and MT-BCS approach," in Proceedings of the 2017 IEEE International Symposium on Antennas and Propagation \& USNC/URSI National Radio Science Meeting, San Diego, CA, USA, July 2017.

[24] M. Carlin, P. Rocca, G. Oliveri, and A. Massa, "Bayesian compressive sensing as applied to directions-of-arrival estimation in planar arrays," Journal of Electrical and Computer Engineering, vol. 2013, Article ID 245867, 12 pages, 2013.

[25] A. Massa, M. Bertolli, G. Gottardi et al., "Compressive sensing as applied to antenna arrays: synthesis, diagnosis, and processing," in Proceedings of the IEEE International Symposium on Circuits \& Systems Meeting, Florence, Italy, May 2018. 


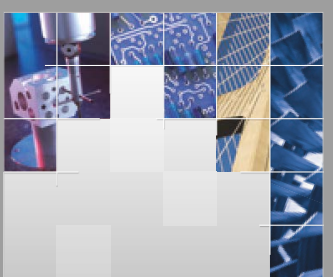

\section{Enfincering}
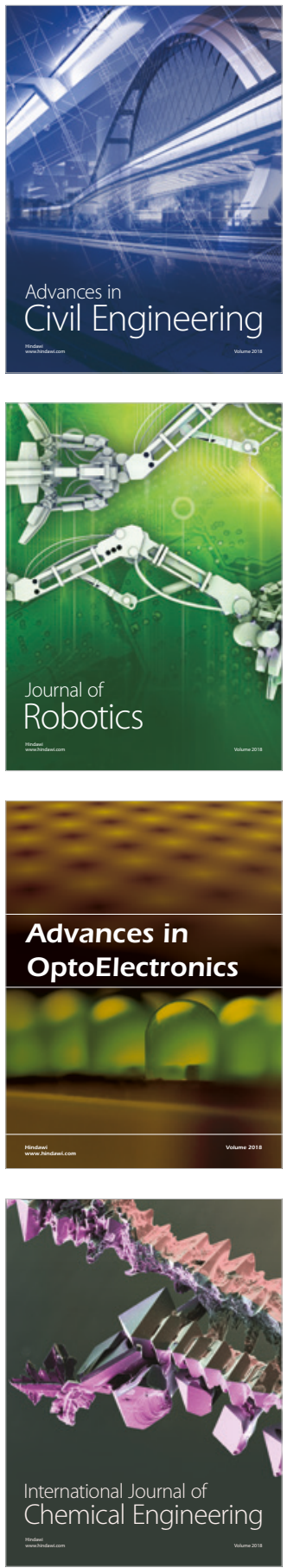

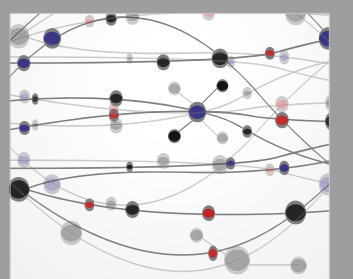

\section{Rotating \\ Machinery}

The Scientific World Journal

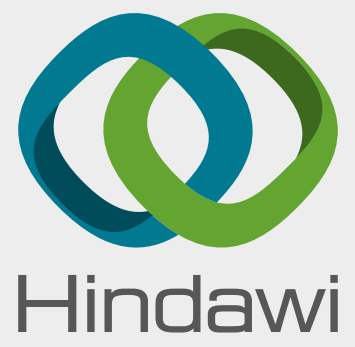

Submit your manuscripts at

www.hindawi.com
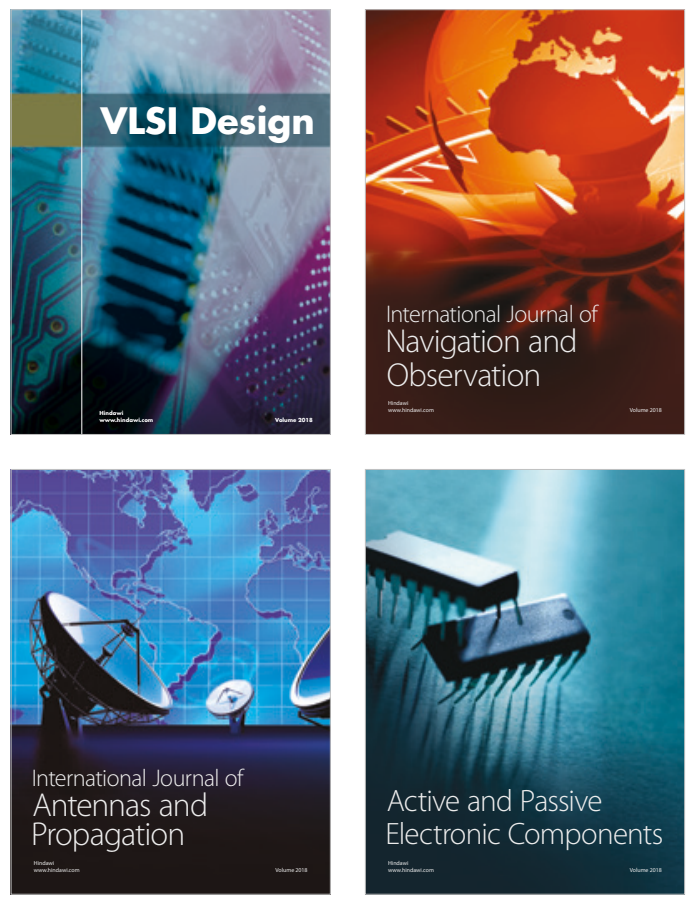
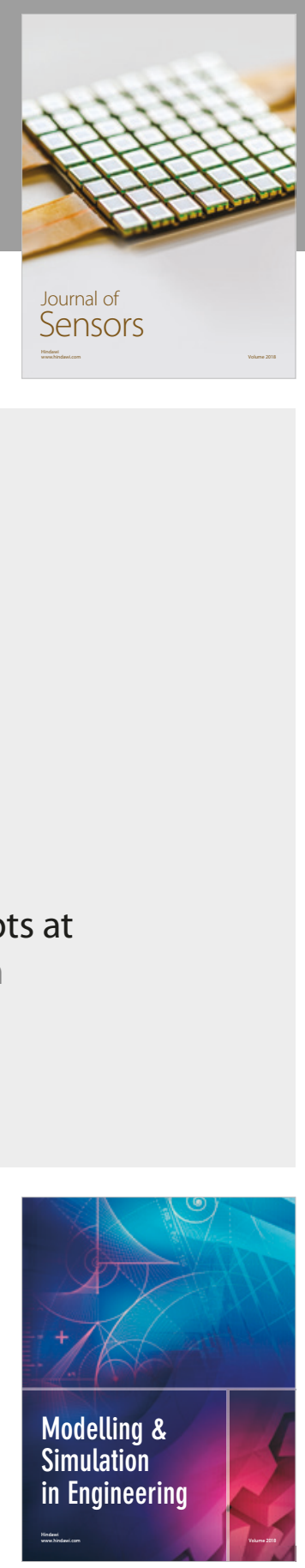

\section{Advances \\ Multimedia}
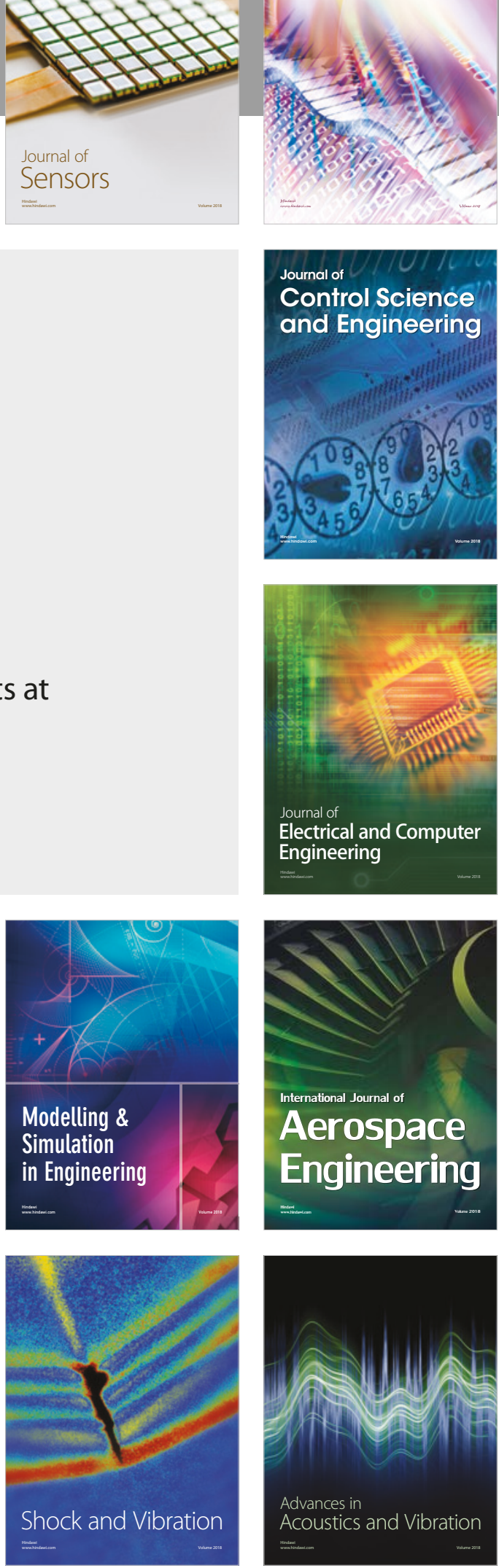